\title{
Texture of electron beam melted Ti-6Al-4V measured with neutron diffraction
}

\author{
M. Neikter ${ }^{*}$, R. Woracek ${ }^{2,3}$, C. Durniak ${ }^{2}$, M. Persson ${ }^{4}$, M.-L. Antti ${ }^{1}$, P. Åkerfeldt ${ }^{1}$, R. Pederson ${ }^{5}$, \\ J. Zhang ${ }^{6}$, S.C. Vogel $^{6}$ and M. Strobl ${ }^{7,3}$ \\ ${ }^{1}$ Luleå University of Technology (Sweden); ${ }^{2}$ European Spallation Source ERIC (Sweden); \\ ${ }^{3}$ Nuclear Physics Institute of the CAZ (Czech Republic); ${ }^{4}$ General Electric Additive Manufacturing (Sweden); ${ }^{5}$ University West (Sweden); ${ }^{6}$ Los \\ Alamos National Laboratory (USA); ${ }^{7}$ Paul Scherrer Institute (Switzerland) \\ *Corresponding author: magnus.neikter@1tu.se, phone: +46 (0)920-492378
}

\begin{abstract}
Texture in materials is important as it contributes to anisotropy in the bulk mechanical properties. Ti-6Al-4V built with the additive manufacturing process (AM) electron beam melting (EBM) has been found to have anisotropic mechanical properties. Therefore, this work has been performed to investigate the texture variations of EBM built Ti-6Al-4V with neutron time of flight (TOF). For the work, samples were produced with different build geometries off-set by 90 degrees. A cast sample was additionally analyzed to investigate the bulk texture of conventionally manufactured material. Microstructural characterization was performed and the cast material was found to have a coarse colony $\alpha$ microstructure, whereas the EBM built material had a finer basket weave microstructure. Overall, the texture of the EBM built material was found to be weak having an multiple of random distribution (MRD) index of $\sim 1$ for the $\alpha$ phase, whilst the cast material possessed more than twice the amount of preferred orientation i.e. MRD 2.51 for the $\alpha$ phase.
\end{abstract}

Keywords: Neutron time-of-flight diffraction; texture; Ti-6Al-4V; electron beam melting; cast

\section{Introduction}

Texture is defined as the amount of preferential crystal orientation in a polycrystalline material (where crystal refers to an individual grain in this context). If the crystal orientations are random, all orientations have the same probability and no texture is present, whereas strong texture signifies that many crystals are oriented in similar directions. For many polycrystalline materials, different crystallographic directions (lattice plane families) have different Young's moduli e.g. ranging from 46.5 to $181 \mathrm{GPa}$ for $\alpha$ titanium [1]. Therefore, strong texture can influence the global mechanical properties, resulting in anisotropy of the bulk material. For the titanium alloy Ti-6Al-4V, two different types of crystal structures can be present: the high temperature body-centered cubic phase $\beta$ and a hexagonal close-packed crystal structure $\alpha$; a phase stable at ambient conditions [2]. Above the $\beta$ transus temperature only the $\beta$ phase is found, but when the temperature is decreased below this temperature (which is $995{ }^{\circ} \mathrm{C}$ for Ti-6Al-4V [2]), the $\beta$ phase transforms into the $\alpha$ phase following the Burgers relationship $\{0001\} \alpha\|\{110\} \beta,<1120>\alpha\|<111>\beta[2,3]$. At room temperature, some residual metastable $\beta$ phase is typically present, depending on the cooling profile, which affects the time for the beta stabilizing vanadium atoms to repartition. The amount of the different phases influences the mechanical properties and for material built with additive manufacturing (AM), anisotropic mechanical behavior has been observed by Lewandowski and Seifi [4]. The reason for this anisotropy has been attributed to defects and their orientations [4]. However, the microstructure is also considered to be a contributing factor. For example, Carrol et al. [5] attributed the anisotropic tensile properties of directed energy deposited Ti-6Al-4V to the columnar $\beta$ grains and the grain boundary $\alpha$ that is found along the prior $\beta$ grain. The columnar prior $\beta$ grains are characteristic for AM built materials, and they are formed during the thermal cycles where the $\beta$ grains grow epitaxially in a columnar fashion along the thermal gradients [6]. There are typically three types of microstructures in additively manufactured titanium, depending on cooling rate. The first alternative is when $\alpha$ phase starts to nucleate along the $\beta$ grain boundary when the temperature is decreased below the $\beta$ transus. Followed by growth of $\alpha$ laths from the $\beta$ grain boundary that is related to Burgers orientation relationship [7]. When numerous $\alpha$ laths grow in a parallel direction they form ' $\alpha$ colonies' i.e. $\alpha$ laths with the same crystal orientation, and the faster the cooling rate the smaller these $\alpha$ colonies become. This type of microstructure is called colony $\alpha$. Albeit, if a faster cooling rate is achieved nucleation of the $\alpha$ phase also occurs at the boundaries of other $\alpha$ colonies [2]. Because the smaller $\alpha$ colonies that nucleate from the $\beta$ grain cannot fill the whole grain. New $\alpha$ laths nucleate by point contact of already existing $\alpha$ laths and they tend to grow perpendicular to the already existing $\alpha$ lath, this alternative is called basketweave. The third alternative for AM built material is the $\alpha$ ' martensite microstructure. This microstructure is formed at very high cooling rates, where no diffusion has time to occur.

Neutron time of flight (TOF) diffraction can be used for measurements of both residual stresses [8] and texture [9,10] in materials. The neutral charge of neutrons leads to high penetration (in the range of $\mathrm{cm}$ ) of many metals [11]. Consequently, texture results from TOF can be regarded as three-dimensional, opposed to texture obtained using electron backscattered diffraction, which renders a more two-dimensional texture due to the low penetration depth of electrons (about $20 \mathrm{~nm}$ [12]). Neutron TOF diffraction has been used to measure the texture of Ti-6Al-4V manufactured from both cast [10] and different AM processes [9]. However, no work (as far as the authors know) have investigated the texture of cast and electron beam melting (EBM) built material, using the same sample dimensions and the current AM process parameters. Therefore, as-built EBM and as-cast Ti-6Al-4V have been investigated in this work with neutron TOF diffraction, to characterize the bulk textures of these materials. The purpose has been to investigate the texture of EBM built Ti-6Al-4V and conventional cast material, and to identify textural variations with respect to EBM build directions i.e. vertical (parallel to the build direction) and horizontal (perpendicular to the build direction).

\section{Material and experiments}

\subsection{Material and microstructural characterization}

Two samples were manufactured using a Q20+ EBM machine from Arcam. This machine was equipped with EBM control version 5.2.52 along with standard process themes version 5.2.24. Important parameters for the different themes are shown in Table 1. The samples were created in the software Materialise Magics with the dimensions $10 \times 10 \times 15 \mathrm{~mm}$ and $10 \times 15 \times 10 \mathrm{~mm}$ (width, length, and height) i.e. vertically and horizontally built respectively. Both samples were positioned in the center of the build envelope with a $2 \mathrm{~mm}$ clearance and built directly on a steel start plate. 
After manufacturing, the samples were removed from the start plate and blasted in an Arcam powder recovery system (PRS) to separate the samples from sintered powder. The spot size used in the EBM process was $0.5 \mathrm{~mm}$.

Table 1: The parameters used to manufacture the EBM samples. The build is categorized into preheat of the material followed by the melting, which is composed of an outer and inner contour and hatching.

\begin{tabular}{llll}
\hline Parameters & Preheat [1 / 2] & Melting [Outer-/Inner- Contour/ Hatch] & Unit \\
\hline Average current & $0 / 13.95$ & - & {$[\mathrm{mA}]$} \\
Focus offset & $100 / 100$ & $6 / 6 / 45$ & {$[\mathrm{~mA}]$} \\
Speed & $40500 / 40500$ & $450 / 450 /-$ & {$[\mathrm{mm} / \mathrm{s}]$} \\
Current & $36 / 45$ & $9 / 9 / 19$ & {$[\mathrm{~mA}]$} \\
Speed function & - & $-/-/ 32$ & {$[-]$} \\
\hline
\end{tabular}

The cast Ti-6Al-4V sample was machined using electric discharge machining (EDM) from a ring of cast material (see Figure 1 a)), where the wall is in the $\mathrm{Z}$ direction. After the EBM process, the samples received the same machining as the cast sample, to obtain the desired shape, which was cylindrical with a diameter of $10 \mathrm{~mm}$ and height of $15 \mathrm{~mm}$ as shown in Figure $1 \mathrm{~b}$ ). Prior to the microstructural characterization, the samples were ground and polished according to conventional methods for titanium alloys. To reveal the microstructure Kroll's etchant was used. To obtain micrographs a Nikon eclipse MA200 light optical microscope (LOM) was used. To measure the $\alpha$ laths the software ImageJ [13] was utilized.

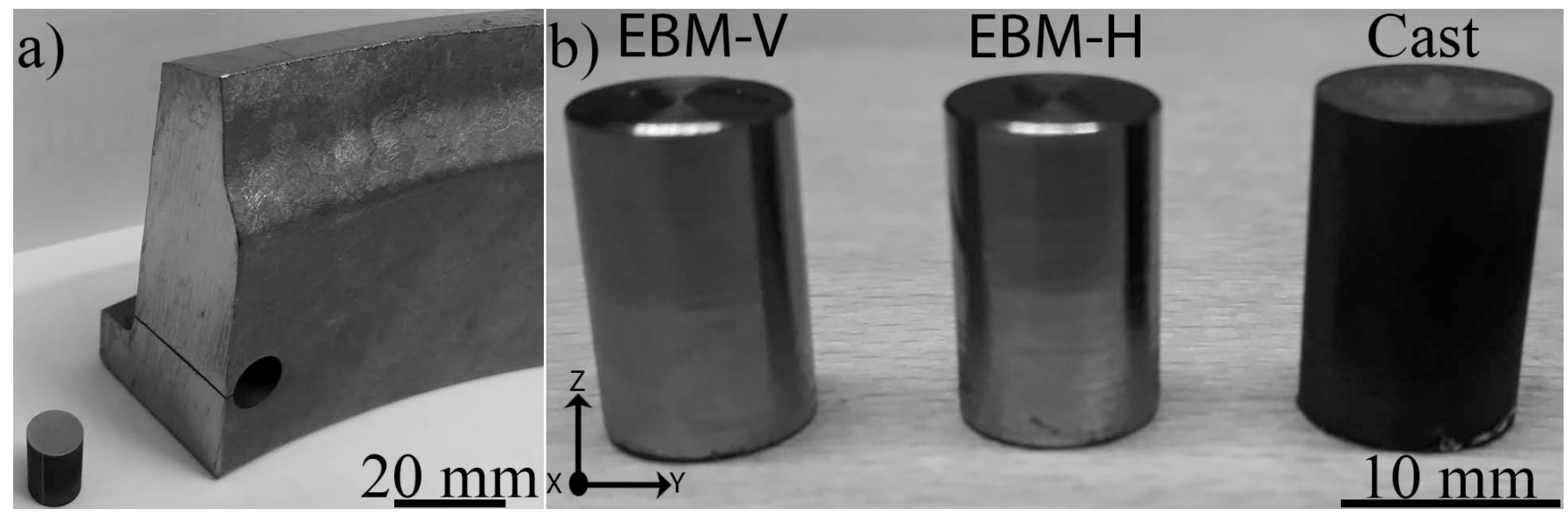

Figure 1: In a) the origin of the cast sample is shown, b) shows a photograph of the samples after machining. $Z$ is the build direction for the vertically built sample, whereas the build direction is found in $\mathrm{X}$ and $\mathrm{Y}$ plane for the horizontal sample. For the cast sample the $\mathrm{Z}$ direction is towards the wall.

\subsection{Bulk texture measurements with neutron time of flight}

Neutron time of flight (TOF) diffraction experiments were performed on the high-pressure-preferred orientation (HIPPO) instrument at Los Alamos Neutron Science Center, USA [14]. The measurements were performed on all three samples. The distance between the samples mounted in the HIPPO instrument and the moderator was 9 meters. The neutron flux with a proton accelerator operating at $120 \mu \mathrm{A}$ was roughly $2.4 \times 10^{7}$ neutrons $\mathrm{cm}^{-2} \mathrm{~s}^{-1}$ [14]. The HIPPO instrument is composed of $1200^{3} \mathrm{He}$ tubes and for this texture experiment the orientations $2 \Theta=0,67.5$ and $90^{\circ}$ were used. The exposure time was set to 20 minutes. The beam spot size was $10 \mathrm{~mm}$ in diameter, ensuring that $\sim 60 \%$ of the sample volume was probed. The measured texture data were analyzed using the MAUD software. The methodology for obtaining the orientation distribution function from the TOF data is explained elsewhere [15]. The texture was refined using least squares Rietveld refinement procedure [16] and the orientation distributions were fitted according to the Entropy-Williams-Imhof-Matthies-Vinel algorithm (E-WIMV) [17] using a resolution for the E-WIMV representation of the orientation distribution function (ODF) of 7.5 degrees.

\section{Results}

\subsection{Microstructure}

The different microstructures of the EBM built and cast materials are shown in Figure 2 a) to f). In the cast material, the microstructure had a colony $\alpha$ morphology, with large $\alpha$ colonies (white dotted line in a) shows an example of an $\alpha$ colony). The $\alpha$ laths were oriented parallel to each other with the same crystal direction. The EBM built material had a fine basketweave microstructure, with the $\alpha$ laths intertwined with one another (Figure $2 \mathrm{e}$ ) and f)). The $\alpha$ lath thickness varied between the two types of material; in the EBM material, the thickness was $0.8 \pm 0.1 \mu \mathrm{m}$ whereas for the cast material it was $3.2 \pm 1.1 \mu \mathrm{m}$. The size of the prior $\beta$ grains was different as well; in b) and c) examples of prior $\beta$ grains in EBM built material are marked with black dotted lines. It can be noted that the prior $\beta$ grains appear circular for horizontally built EBM (EBM-H) and columnar for vertically built EBM (EBM-V). For the cast material, the prior $\beta$ grains were larger than for the EBM material, being in the range of several $\mathrm{mm}$. These prior $\beta$ grains were easily distinguished due to thick grain boundary $\alpha$, whereas in the EBM material this was not the case. Furthermore, in the EBM material several pores were present as well (examples are indicated by black arrows in Figure $2 \mathrm{c}$ )). 


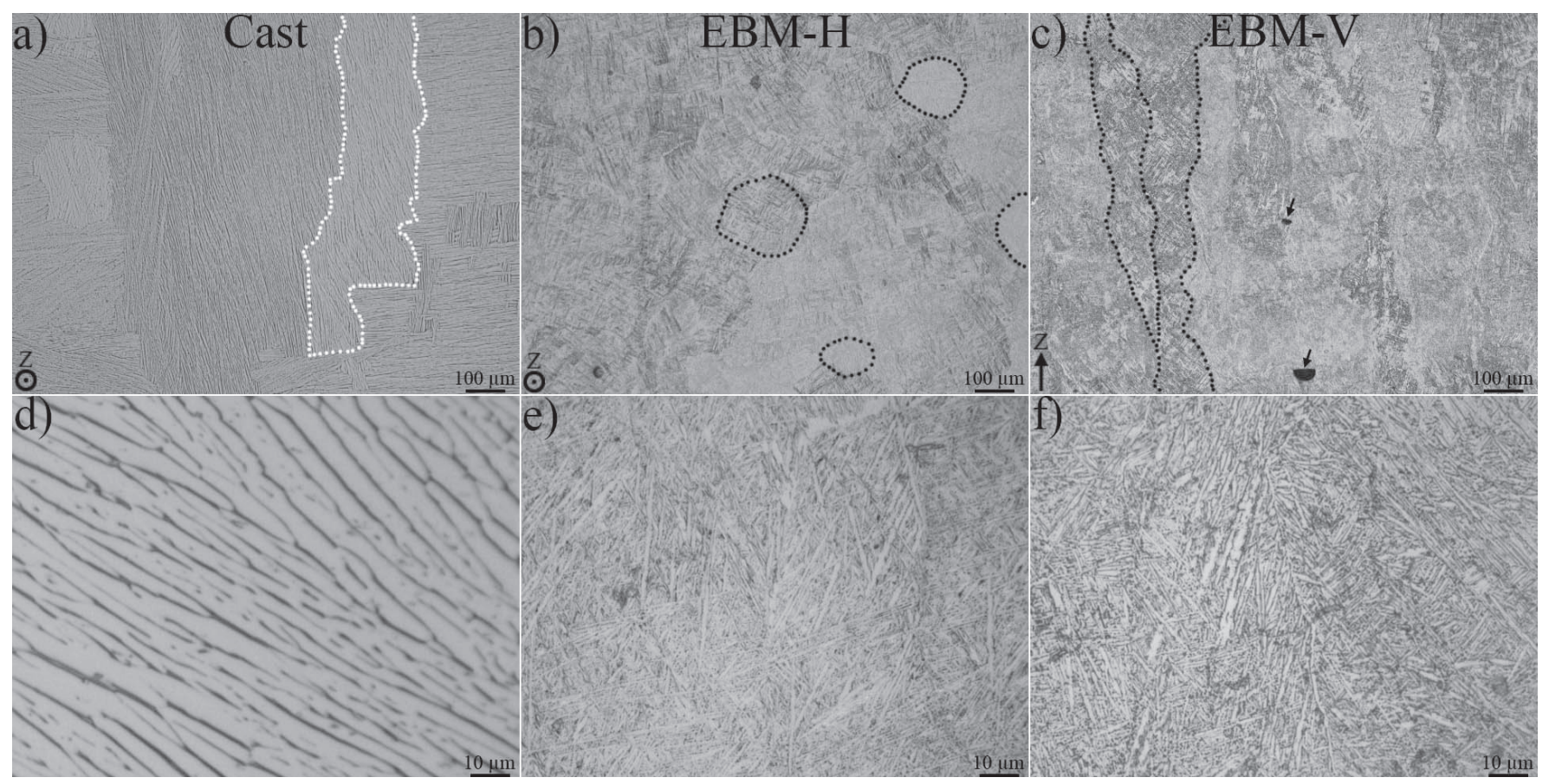

Figure 2: Low magnification micrographs of cast material with large $\alpha$ colonies (a), followed by EBM-H (horizontal, b), and EBM-V (vertical, c). The black dotted lines in b) and c) exemplifies prior $\beta$ grains. The black arrows show pores. In d) to f) higher magnification micrographs of cast, EBM-H and EBM-V are shown respectively.

\subsection{Pole figures}

Figure 3 shows the pole figures recalculated from the orientation distribution function (ODF) derived from neutron diffraction experiments. In Table 2 the multiple of random distribution (MRD) index is shown, i.e. the average bulk texture. The cast material had a relatively strong texture, being MRD of 2.51, whilst the vertical and the horizontal EBM sample had a weak texture of MRD $\sim 1$. The strongest texture was observed in the basal plane for all three displayed slip planes i.e. basal $(0002)$, prismatic 1010$)$ and pyramidal (1011) (an illustration of the slip planes is shown in the bottom right of Figure 3). For the pyramidal and prismatic planes there was no large difference in texture within each sample. The pole figures have several strong texture components (the black arrows in Figure 3 exemplifies stronger texture components) e.g. the build direction ( $Z$ ) for the prismatic plane. The average texture was stronger for the $\beta$ compared to the $\alpha$ phase for all the investigated samples.

Table 2: The $\alpha$ and $\beta$ phase fraction, followed by the max MRD values for the $\alpha$ phase for the three slip planes, and the texture MRD index, i.e. average texture, for the $\alpha$ and $\beta$ phase.

\begin{tabular}{|c|c|c|c|}
\hline & Cast & EBM-V & EBM-H \\
\hline$\alpha$ phase fraction (at\%) & $96.7 \pm 6.5$ & $97.3 \pm 4.4$ & $97.2 \pm 4.7$ \\
\hline$\beta$ phase fraction (at\%) & $3.3 \pm 0.2$ & $2.7 \pm 0.1$ & $2.8 \pm 0.1$ \\
\hline Max MRD basal $(\alpha)$ & 3.96 & 1.51 & 1.33 \\
\hline Max MRD pyramidal $(\alpha)$ & 2.08 & 1.12 & 1.11 \\
\hline Max MRD prismatic ( $\alpha)$ & 1.95 & 1.17 & 1.17 \\
\hline Texture MRD index $(\alpha)$ & 2.51 & 1.03 & 1.03 \\
\hline Texture MRD index $(\beta)$ & 3.54 & 1.36 & 1.21 \\
\hline
\end{tabular}




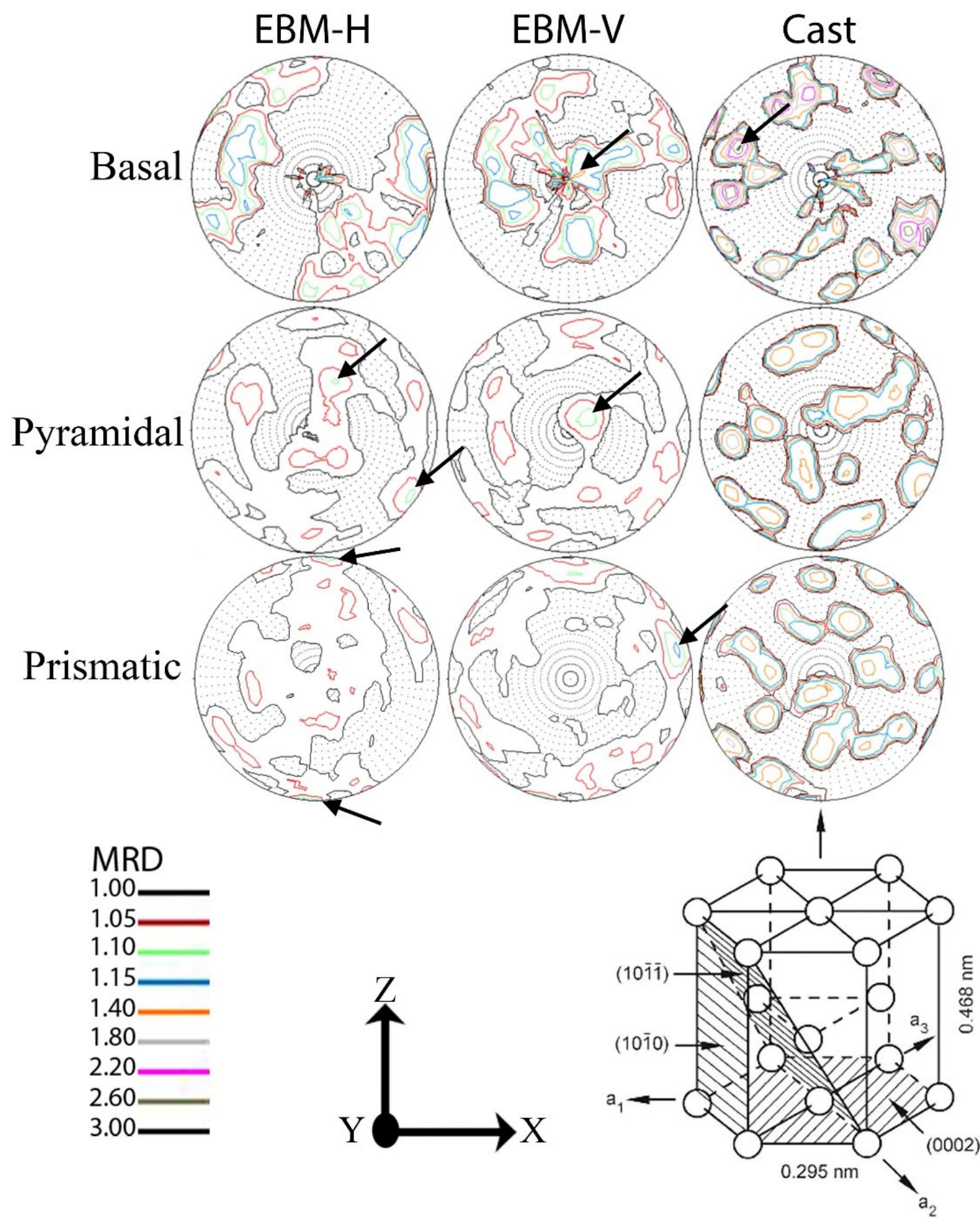

Figure 3: Pole figures obtained from neutron TOF diffraction measurements for the horizontal (H), vertical (V) EBM and cast samples generated with PF-plot (written by C. Umlauf and K. Ullemeyer in 2006). They show the texture of the basal, prismatic, and pyramidal slip planes for the $\alpha$ phase. Arrows indicate strong texture components. In the bottom right corner, the investigated slip planes are illustrated within a hexagonal close packed crystal structure.

\section{Discussion}

In this work neutron TOF diffraction was utilized to measure the texture. The reason for choosing this texture measurement method instead of e.g. EBSD, was due neutrons high penetration depth. Thus, the bulk texture of the EBM built and cast material could be investigated. For the two types of investigated material two different microstructures were observed where the $\alpha$ lath size is a good indication of the cooling rates. The EBM built material experienced a relatively fast cooling rate, as the thinner the $\alpha$ laths are the faster the cooling rate has been $[2,18]$, while for the cast material the $\alpha$ laths were coarse. The reason for this difference in cooling rates originates from the manufacturing processes. The casting process had in this scenario a slow cooling rate (cooling rate depends on batch size, etc.). In the EBM process, the build platform is kept at a temperature $\geq 600{ }^{\circ} \mathrm{C}$, and small volumes of material are melted at the same time. The small melt pools render a relatively fast cooling. Apart from the $\alpha$ lath size, another difference between the investigated material is the prior $\beta$ grain size and shape. In the investigated cast material, they are very large, whereas in EBM they are thinner and have an elongated shape. The thin prior $\beta$ grain size of the EBM material does not allow the $\alpha$ colonies to become large, as an $\alpha$ colony cannot become larger than its parent $\beta$ grain. The small $\alpha$ colonies and prior $\beta$ grains explains the comparably weaker texture of the EBM material. The same trend in prior $\beta$ grain size was observed by Neikter et al. [9] in regard to microstructure, who investigated both EBM and laser metal wire deposited (LMwD) Ti-6Al-4V. The LMwD built material had large prior $\beta$ grains, which rendered in a stronger texture when compared to the EBM material. The LMwD material in that work and the cast material in this work had roughly the same maximum texture, 3.49 MRD for LMwD whereas an MRD of 3.96 for the cast material, both for the basal plane. In addition, the EBM material in this work and in [9] had 
the same texture, 1.56 and 1.51 (vertical section) respectively for the basal plane. In the pole figures, there seems to be an orientation difference of 90 degrees between horizontally and vertically built material e.g. the strongest texture maximum for the prismatic plane is found in the $\mathrm{Z}$ direction for the horizontal sample, whereas the maximum for the vertical sample is found in the X direction. Thus, by turning the pole of EBM-H by 90 degrees clockwise roughly the same pole figure as EBM-V is obtained. The reason for not being oriented exactly 90 degrees could be related to that the prior $\beta$ grains are not orientated straight either. In the EBM material, they are shifted slightly toward the moving heat source, thus possibly influencing the orientation of the pole figures. Finally, it was shown that the $\beta$ texture was stronger than the $\alpha$ texture, for both the cast and EBM material. This is possibly due to the lower amount of $\beta$ phase at room temperature, thus lower statistics. In [9], the lower amount of statistics of the EBSD texture measurement resulted in stronger texture.

\section{Conclusions}

In this work, the texture of electron beam melted and cast Ti-6Al-4V has been investigated using neutron time of flight (TOF) diffraction. Two types of electron beam melted (EBM) material have been investigated, built in perpendicular directions. In addition, microstructural characterization of the material has been conducted using light optical microscopy. The texture results have been linked to the microstructures of the two types of investigated material and the following conclusions could be drawn:

- The cast material had a coarse colony $\alpha$ microstructure, whereas the EBM material possessed a finer basketweave microstructure.

- The investigated cast material had a relatively strong texture, having multiples of random distribution index of 2.51 .

- The electron beam melted material had a relatively weak texture, having multiples of random distribution index of $\sim 1$.

- The stronger texture of the cast material was correlated to its larger prior $\beta$ grains and $\alpha$ colonies, whereas the opposite was true for the EBM material.

- The $\beta$ phase showed a stronger texture than the $\alpha$ phase.

\section{Acknowledgments}

The authors would like to thank the Los Alamos Neutron Science Center, Los Alamos, USA for providing the neutron beam time (proposal no. LC2019-7913-A) at the HIPPO neutron TOF diffraction instrument. Thanks to Prof. Luca Lutterotti, author of the Rietveld software MAUD, making it possible to analyze the texture data. For financial support, the author M.N. would like to acknowledge Nationellt rymdtekniskt forskningsprogram, the EU funded Space for innovation and growth, the Graduate School of Space Technology at Lulea University of Technology and the Wallenberg Foundation. R.W. and M.S. would like to acknowledge the support from OP RDE.MEYS, under the project "European Spallation Source participation of the Czech Republic - OP”, Reg. No. CZ.02.1.01/0.0/0.0/16013/0001794.

\section{References}

[1] D. Tromans. , Int.J.Res.Rev.Appl.Sci 6(4) (2011) 462-483.

[2]G. Lütjering, J.C. Williams. Titanium, Second ed. (2007).

[3] N. Sridharan, A. Chaudhary, P. Nandwana, S.S. Babu, JOM 68(3) (2016) 772-777.

[4] J.J. Lewandowski, M. Seifi, Annual Review of Materials Research 46 (2016) 151-186.

[5] B.E. Carroll, T.A. Palmer, A.M. Beese, Acta Materialia 87 (2015) 309-320.

[6] M. Neikter, P. Åkerfeldt, R. Pederson, M. Antti et al, Mater Charact 143 (2018) 68-75.

[7] D. Bhattacharyya, G.B. Viswanathan, H.L. Fraser, Acta Materialia 55(20) (2007) 6765-6778.

[8] T. Maimaitiyili, R. Woracek, M. Neikter, M. Boin et al, Materials 12(4) (2019) 667.

[9] M. Neikter, R. Woracek, T. Maimaitiyili, C. Scheffzük et al., Additive Manufacturing 23 (2018) 225-234.

[10] G.C. Obasi, R.J. Moat, D.L. Prakash, W. Kockelmann et al, Acta Materialia 60(20) (2012) 7169-7182.

[11] U.F. Kocks, C.N. Tome, H. Wenk, Cambridge University, Cambridge (1998).

[12]A.J. Schwartz, M. Kumar, B.L. Adams, D.P. Field. Electron backscatter diffraction in materials science, (2009).

[13] M.D. Abràmoff, P.J. Magalhães, S.J. Ram, Biophoton Int 11(7) (2004) 36-42.

[14] H. Wenk, L. Lutterotti, S. Vogel, Nuclear Instruments and Methods in Physics Research Section A: Accelerators, Spectrometers, Detectors and Associated Equipment 515(3) (2003) 575-588.

[15] H. Wenk, L. Lutterotti, S.C. Vogel, Powder Diffraction 25(3) (2010) 283-296.

[16] H. Rietveld. , Journal of applied Crystallography 2(2) (1969) 65-71.

[17] L. Lutterotti, D. Chateigner, S. Ferrari, J. Ricote, Thin Solid Films 450(1) (2004) 34-41. 
[18]M. Neikter, P. Åkerfeldt, R. Pederson and M-L. Antti. IOP Conference Series: Materials Science and Engineering: IOP Publishing 258(1) (2017). 